International Agency for Research on Cancer (IARC)

1976 Annual Report. (Pp. 159; illustrated; Sw.fr. 12, \$4.80.)

IARC Monograph on the Evaluation of Carcinogenic Risk of Chemicals to Man. Volume 13: Some Miscellaneous Pharmaceutical Substances. (Pp. 256; Sw.fr. 30, \$12.)

Volume 14: Asbestos. (Pp. 108; Sw.fr. 14, \$6.) Lyon: International Agency for Research on Cancer. 1977. (Available through HMSO, London).

\section{The first 10 years of the IARC}

The photograph on the front of IARC Annual Reports of the 5-year-old offices and laboratories at Lyon gives a false impression of great size. In fact, by comparison with other cancer research organisations, the IARC with less than 200 staff and a budget in 1976 of only just over six million US dollars is small, and this needs to be borne in mind when assessing what it has achieved during the first 10 years of its existence.

The emphasis of the IARC's efforts has appropriately been on environmental carcinogenesis against a background of epidemiological and geographical cancer incidence data. Backing this up has been the award during the 10 years of 183 research training fellowships and 228 travel fellowships. It is difficult to assess how much of what the IARC has done would not have been done if it had not come into existence as a separate organisation. Work along the same lines was being funded by the British Empire Cancer Campaign (now the Cancer Research Campaign) and by numerous national bodies in various countries before the IARC appeared on the scene. Even so, the coordination of such efforts by the IARC has been of very real value and undoubtedly hastened progress.

The series of IARC monographs on the Evaluation of Carcinogenic Risk of Chemicals to Man, of which volumes 13 and 14 have just been published, have been of value in several ways. Firstly, their production has brought together scientists from different countries to discuss the quality of experimental and epidemiological data relevant to the evaluation of carcinogenic risk. As a consequence some of the scientists concerned have appreciated for the first time the equivocal nature of some of the evidence. This in turn has led to further work and to a general raising of standards of experimentation. At the same time the monographs have provided useful sources of reference for regulatory bodies, cancer research scientists, and scientists involved in determining the likely safety of drugs, pesticides, food chemicals, and industrial processes. The majority of actual evaluations of chemicals provided in the monographs are less helpful than they might have been because, where there are no human data (as is usually the case), no attempt is made to judge whether the results of animal tests indicate serious, intermediate, or negligible hazard. If most of the substances reviewed had been cleared of suspicion of cancer hazard, this would not have mattered so much, but this is not the case. For instance, of the 17 drugs considered in volume 13, 10 are deemed to have given definitely positive results in animals and two doubtfully positive results in animals, three-phenacetin, oxymetholone, and phenytoin-are designated as being carcinogenic to man, and a further three are not evaluated at all. None is cleared of suspicion. Clinicians would obviously want to know whether there is a cancer risk from any of these drugs serious enough to influence their prescribing patterns. In practice the monograph provides no useful guidance on this. In my opinion, it is unlikely that any of the drugs reviewed constitute a serious cancer hazard, and it is a pity that this is not stated clearly either in the introduction to the volume or in the press release that accompanied its publication. On the other hand, the press release does include a qualifying statement which is so full of wisdom as to be worth reproducing in toto:

'When evaluating drug-cancer relationships, the following should be considered in addition to the possibility that the drug itself may cause cancer: (1) the pathological condition may predispose to cancer; (2) the cancer may predispose to the pathological condition; (3) the pathological condition or the treatment may increase the chance of diagnosis of cancer but not cause an increase in its incidence; (4) some third factor (eg, a genetic factor) may cause both the pathological condition and cancer, which are not otherwise related; (5) the pathological condition and the cancer may be

considered phases of the same proces름 (6) patients may be exposed to more that one drug either sequentially or in comb nation; (7) patients given the drug may, a consequence, survive longer.'

Volume 14 of the monographs of asbestos is open to the criticism that man $\vec{\phi}$ of the evaluators were evaluating their own work or that of others present at thes meeting. This mitigates against impar tiality. On the other hand, in such a high $\$$ technical and complex area it is probablyo essential to have a quorum of experienceg experts present. Few can now be unawaile of the seriousness of the cancer hazard which industrial exposure to asbestos dust entails. Equally, no one can realistically suggest that the use of asbestos should be abandoned and so far animal experiments have been of only limited value in provict ing answers to urgent practical problen such as the determination of the relative risks from different forms of asbestos and whether there is any cancer risk from the use of asbestos filters in beer manufacture or sheets of asbestos on domestic ironing boards.

All in all, congratulations are certain due to the IARC for its achievements date, and the indications are that $\mathrm{i}$ importance will increase provided that resists domination by the attitudes an policies that prevail in the United States?

F. J. C. R胳 